\title{
Analysis of Multi-objective Decision Problems in Humanitarian Supply Chains
}

\author{
Takeo Kobayashi \\ Production Division, \\ RICOH COMPANY LTD., 1005 Shimo-ogino, Atsugi, Kanagawa, 243-0298, Japan \\ Email: takeo.tk.kobayashi@nts.ricoh.co.jp \\ Yacob Khojasteh \\ Graduate School of Global Studies, \\ Sophia University, 7-1 Kioi-cho, Chiyoda-ku, Tokyo, 102-8554, Japan \\ Email: khojast@sophia.ac.jp \\ Yasutaka Kainuma \\ Graduate School of Systems Design, \\ Tokyo Metropolitan University, 6-6 Asahigaoka, Hino, Tokyo 191-0065, Japan \\ Email: kainuma@tmu.ac.jp (Corresponding Author)
}

\begin{abstract}
In humanitarian supply chains, there is a need to consider how to distribute the limited relief supplies that have not been prepared sufficiently to each shelter. Different from commercial supply chains that prioritize cost reduction, in humanitarian supply chains, it is important to consider not only reducing the distribution cost but also how to deliver goods quickly to satisfy the demands of shelters. Three metrics have been proposed for humanitarian supply chains: efficiency, equity, and efficacy. The objective of this paper is to define the metric of efficacy to minimize distribution time to each shelter considering the relief goods arrival. Under the situation after the disaster, to supply relief goods agilely and fairly, it is necessary to take account the weight of each metric adequately. We formulate a multiobjective mathematical model by the multiple metrics and carry out the optimization by using the model. The results show that the proposed multi-objective model is very promising in dealing with complicated humanitarian supply chains. Additionally, we show the deviation between the result of each metric and the multi-objective evaluation.
\end{abstract}

Keywords: humanitarian supply chains, disaster relief operations, metrics of humanitarian supply chains, multi-objective optimization

\section{INTRODUCTION}

The Great East Japan Earthquake hit the north-eastern parts of Japan (the Töhoku region) on March 11 ${ }^{\text {th }}, 2011$ (Holguin-Veras et al., 2014). The scale of the earthquake was the largest in recorded history in Japan. It disrupted both domestic and global supply chains. It caused significant disruptions in the supply chains, so that many companies and their part suppliers - often a single source - were unable to deliver products at expected volumes for several months. Also, there were various problems in relief supply distributions. Moreover, the 2016 Kumamoto Earthquake hit Kumamoto and Oita Prefectures in Japan (the Kyushu region) on April 14 $4^{\text {th }}, 2016$. In either case of disaster, relief goods were supplied up as far as disaster-affected prefecture. But, victims of the disaster could not receive enough amount of the relief goods. As a main problem, the distribution was inequity for each shelter and the relief goods were not supplied appropriately. Therefore, in disaster-relief activity, it is necessary to consider humanitarian logistics, which is different from commercial case in some aspects.

Humanitarian logistics is a field of logistics which focuses on arranging the warehousing and delivery of materials during complicated emergencies or natural disasters to individuals and areas which have been affected (CILT, 2013). It is in fact a critical element of an effective disaster relief process. Apte (2009) defined humanitarian logistics as a special branch of logistics which manages response supply chain of critical supplies and services with challenges such as demand surges, uncertain supplies, critical time windows in face of infrastructure vulnerabilities, and vast scope and size of the operations. Different from commercial logistics that prioritizes cost reduction, in humanitarian logistics, it is important to consider not only reducing the distribution cost but also how to deliver goods quickly to satisfy the demands of shelters. Therefore, in humanitarian logistics, there is a need to consider how to distribute the limited relief supplies that have not been prepared sufficiently to each shelter. In a view of this situation, three metrics for humanitarian logistics have been proposed. They are efficiency, equity, and efficacy. Efficiency deals with minimizing the distribution cost, which is a basic attitude in the commercial logistics. Equity means supplying goods equally to each shelter without any variance. However, Efficacy deals with a rapid distribution of supplies to all shelters. The way of distributing supplies becomes different by prioritizing the three metrics of humanitarian support. In this paper, we particularly focus on 
the efficacy metric because the definition of this metric is varied. The objective is to define and address the metric of efficacy in humanitarian logistics to minimize distribution time to each shelter considering the relief goods arrival. Moreover, we show the characteristic of the metric of efficacy by comparing it with the other two metrics. Furthermore, we consider more effective supplying method of relief goods according to the post-disaster situation by formulating a multi-objective mathematical model.

The remainder of this paper is organized as follows. Section 2 presents a brief literature review on management of operations in humanitarian supply chains. Metric of efficacy is defined in Section 3. A mathematical model is also developed in this section. Numerical experiments in the case of the Great East Japan Earthquake are presented in Section 4. Discussions and conclusions are presented in Section 5.

\section{LITERATURE REVIEW}

Regarding relief supply distribution in humanitarian aid, studies in the literature have suggested that humanitarian supply chain design must differ from that of commercial supply chain in order to perform more effective and efficient operations considering cost and speed (Tomasini and Van Wassenhove, 2009). Specifically, Özdamar et al. (2004) investigated multi-relief goods network flow and Haghani and Oh (1996) addressed the multi-relief goods network flow problem with respect to time constraints.

In humanitarian logistics, the three metrics of efficiency, equity, and efficacy are commonly used as primary factors determining the success of an operation (Huang et al., 2012). There are many challenges in distributing what is needed, when it is needed, and where it is needed, in addition to meeting supply and demand needs, and therefore, these three metrics have accordingly been proposed for the construction of a practicable model (Kato and Kubo 2012). To distinguish these metrics, Tzeng et al. (2007) proposed three objectives: economy, effectiveness, and satisfaction. In the both abovementioned researches (Huang et al., 2012; and Kato et al., 2012), there were existing common approaches to quantify efficiency and equity. Efficiency is considered to be a measure of distribution costs with respect to time and/or cost. Huang et al. (2012) highlighted that distribution cost is a direct reflection of distribution time, and Kato and Kubo (2012) pointed out that distribution cost is a combined reflection of inventory holding costs, the costs of the supply point, etc. Equity, or equitable distribution, is considered a measure of unsatisfied demand of victims, achieved by using a disutility function. In particular, the disutility function is represented by unsatisfied demands in the both research investigations.

In contrast with these metrics, efficacy refers to the rapid distribution. Huang et al. (2012) proposed a model to minimize the demand-weighted arrival times to achieve a rapid distribution. On the other hand, Kato and Kubo (2012) proposed linking the rapid distribution with minimization of out-of-stock costs as a means of increasing efficacy. As such, these studies considered not only efficiency and equity, but also efficacy in the context of humanitarian logistics. However, their perceptions of what efficacy means are different, though rapid distribution is highly regarded in both. Furthermore, Huang et al. (2015) used factors such as lifesaving utility, delay cost, and equality. In their proposed model, they considered the aspects of cost and equality as well as the lifesaving as a humanitarian aid, which was distinct from the metric of efficacy.

Regarding the quick response in a disaster, Pujawan et al. (2009) proposed four principles, and in a later study two more principles were proposed by Kabata and Kainuma (2013). These six principles of a supply chain in a disaster are information visibility, coordination, accountability, professionalism, agility, and network design. It should be noted that coordination is a strategic response for relationships amongst organizations in the supply chain (Xu and Beamon, 2006). Kabata and Kainuma (2013) suggested the need for establishing borderline push-type and pull-type deliveries from a network design aspect as well as a rapid response from an agility aspect. Further, with regard to relief supply distribution of humanitarian logistics, distributing relief goods in two stages (before and after the disaster occurrence) accounts for both costs and smooth delivery (Chakravarty, 2014).

Concerning equity, in order to deliver relief goods fairly to every shelter, the concept of fulfillment rate for demands may be incorporated to achieve maximum equity (Soeta et al., 2015). In addition, Balcik et al. (2008) considered unsatisfied demand using penalty costs.

There are several studies regarding the relief supply distribution in disasters. See, for examples, Bealt et al. (2016), Rezaei-Malek et al. (2016), Özdamar and Ertem (2015), Hoyos et al. (2015), Diaz et al. (2015), L'Hermitte et al. (2015), Chou et al. (2014), Rath and Gutjahr (2014), Özdamar and Demir (2012) and Lin et al. (2011). Particularly, some studies focused on cost, rapid distribution, and equity for humanitarian logistics. However, there are not many studies that have employed the term "efficacy." The metric of efficacy for relief supply distribution is not completely covered by the metric of efficiency and equity. Therefore, this study aims to define and formulate a means for calculating the metric of efficacy in relief supply distribution distinctly from the metrics of efficiency and equity.

\section{METRIC OF EFFICACY}

\subsection{Definition of Efficacy}

Excluding efficiency and equity, with a special consideration of periods after occurring the disaster, we suggest a model so that the rapid delivery of relief goods to the shelters to be effective distribution. In addition, in terms of the specific disaster logistics, the needs pertaining to "what," "when," "how much," and "where" must be identified. Immediately after the disaster, it is very important to deliver rapidly so as not to waste distribution time and that the victims get the relief goods they specifically need. From these two ideas, different from efficiency and equity, efficacy is determined to be a measure of distribution time. Therefore, we define efficacy as minimizing the amount of goods that cannot be delivered during the distribution time. 


\subsection{Logistics Model}

In the basic logistics model of a disaster, relief goods are usually supplied to shelters via a supply source consisting of a first stockyard of prefectural level and a second stockyard of municipality level. In the Great East Japan Earthquake, relief goods were not delivered as planned from the second stockyard to shelters. In this paper, we focus on the distribution from the second stockyard to the shelters, which represents the last-mile distribution in humanitarian logistics.

Since in our model, we considered the last-mile distribution from second stockyard to shelters, we set the second stockyard as one place and the shelters as $N$ places, where $N$ is a finite natural number. Additionally, we assume that vehicles are used as the mode of transportation, where one vehicle goes to one shelter. As in a disaster, many relief goods are needed, we use a multi-relief goods approach in this study.

\subsection{The Model}

For the mathematical description of the model the following notation is introduced.

\section{Notation}

$\begin{array}{cl}\text { Indices } & \\ i & \text { Shelter index, } i=1,2, \ldots, N \\ p & \text { Relief goods index, } p=1,2, \ldots, P\end{array}$

Parameters

$d_{i p} \quad$ Demands of relief goods $p$ at shelter $i$

$t_{i} \quad$ Distribution time from stockyard to shelter $i$

$K \quad$ Number of available vehicles

$Q \quad$ Capacity per vehicle

$S_{p} \quad$ Available supplies of relief goods $p$ from stockyard

$w_{p} \quad$ Weight of relief goods $p$ by the unit

Decision variables

$x_{i p} \quad$ Amount of relief goods $p$ to shelter $i$

$k_{i} \quad$ Number of vehicles to shelter $i$ follows.

The formulation of the model is developed as

$$
\text { Minimize } \quad \sum_{i} \sum_{p}\left(d_{i p}-x_{i p}\right) \frac{1}{t_{i}}
$$

subject to

$$
\begin{array}{cc}
x_{i p} \leq d_{i p} & \forall i, p \\
\sum_{i} x_{i p}=S_{p} & \forall p \\
\sum_{p} x_{i p} w_{p} \leq Q k_{i} & \forall i
\end{array}
$$

$$
\begin{array}{lc}
\sum_{i} k_{i} \leq K & \\
x_{i p} \geq 0 & \forall i, p \\
k_{i} \text { integer } & \forall i
\end{array}
$$

Considering the goal of effective utilization of distribution time, the objective function indicates the value of the amount of goods that cannot be delivered during the distribution time. Equation (2) represents the upper limit of the amount of delivery to each shelter, in other words the demand of the shelter. Equation (3) is the constraint of the supplies available for delivery. Equation (4) is the constraint of the vehicle capacity. Equation (5) is the constraint of the number of available vehicles. Equation (6) shows nonnegativity, and equation (7) shows that the number of vehicle is non-negative and integer.

\section{NUMERICALEXPERIMENTS}

\subsection{Numerical Experiments}

The Great East Japan Earthquake hit the north-eastern parts of Japan (the Tohoku region) on March $11^{\text {th }}, 2011$, the scale of which was the largest in recorded history in Japan. After the disaster, in addition to the significant disruptions in supply chains, there were various problems in relief supply distribution. As a main problem, the distribution was inequity for each shelter and the relief goods were not supplied appropriately.

In this study, to carry out numerical experiments, we use the number of evacuee data of Soma City in Fukushima Prefecture (Soeta et al., 2015). We set available supplies to be 100 units of rice, 590 units of snacks, 290 units of water, 250 units of food, and 190 units of blankets. Demands of each shelter were calculated based on the number of evacuees. We set the Soma City Hall to be the second stockyard. The required traveling time by a vehicle from the second stockyard to each shelter, and the demands of each shelter are given in Table 1. We surveyed using Google Maps to determine the required time for a vehicle to travel from the second stockyard to each shelter. We assumed that the number of available vehicles is 15 , and the capacity of one vehicle is $750 \mathrm{~kg}$. The Gurobi Optimizer 6.5.0 was used to solve the optimization problem.

In addition, to evaluate the metric of efficacy, we also investigated the efficiency and equity models. Efficiency is considered to be a measure of minimization of distribution cost. Therefore, we solve the problem for the minimum time of the whole distribution as shown in statement (8).

$$
\text { Minimize } \quad \sum_{i} k_{i} t_{i}
$$

Statement (8) is the objective function that indicates efficiency, minimizing the distribution costs. The constraints are the same as those given in equations (2) through (7). 
Equity is considered by equitable distribution (Soeta et al., 2015; and Tzeng et al., 2007). By using the parameter of minimum fulfillment rate of relief goods, we minimize the non-fulfillment rate using equation (9).

\section{Parameter}

$m f_{p} \quad$ Minimum fulfillment rate of relief goods $p$

Minimize

$$
\begin{array}{cc}
\sum_{p}\left(1-m f_{p}\right) & \\
\frac{x_{i p}}{d_{i p}} \geq m f_{p} & \forall i, p
\end{array}
$$

Statement (9) is the objective function that indicates equity, minimizing the non-fulfillment rate. The constraints are the same as those given in equations (2) through (7) and equation (10) which indicates the constraint of the fulfillment rate.

\subsection{Results}

The results of the numerical experiments based on our proposed metrics of efficacy, efficiency, and equity are shown in Figures 1 to 3, respectively. They show the fulfillment rates indicating the ratios of the amount of delivery to the amount of demand.

Table 1 Demands of each Relief Goods in each Shelter

\begin{tabular}{lcccccc}
\hline \multicolumn{1}{c}{ Shelter (the number of evacuees) } & Rice & Snack & Water & Foods & Blanket & Time(min) \\
\hline Sports Arena SOMA (260) & 13 & 78 & 33 & 33 & 26 & 1 \\
The 1st Nakamura Elementary School (450) & 23 & 135 & 57 & 57 & 45 & 1 \\
Hamanasu Welfare Center (500) & 25 & 150 & 63 & 63 & 50 & 5 \\
Yamakami Community Center (22) & 2 & 7 & 3 & 3 & 3 & 8 \\
Yawata Elementary School (142) & 8 & 43 & 18 & 18 & 15 & 8 \\
Roujin Ikoinole (28) & 2 & 9 & 4 & 4 & 3 & 9 \\
Somahigashi High School (489) & 25 & 147 & 62 & 62 & 49 & 9 \\
litoyo Elementary School (150) & 8 & 45 & 19 & 19 & 15 & 10 \\
Koyo Junior High School (460) & 23 & 138 & 58 & 58 & 46 & 10 \\
Nittaki Community Center (49) & 3 & 15 & 7 & 7 & 5 & 13 \\
$\quad$ Total & 132 & 767 & 324 & 324 & 257 & 74 \\
\hline
\end{tabular}

\section{$\square$ Rice $\square$ Snack $\square$ Water $\square$ Food $\square$ Blanket}

Sports Arena SOMA (1)

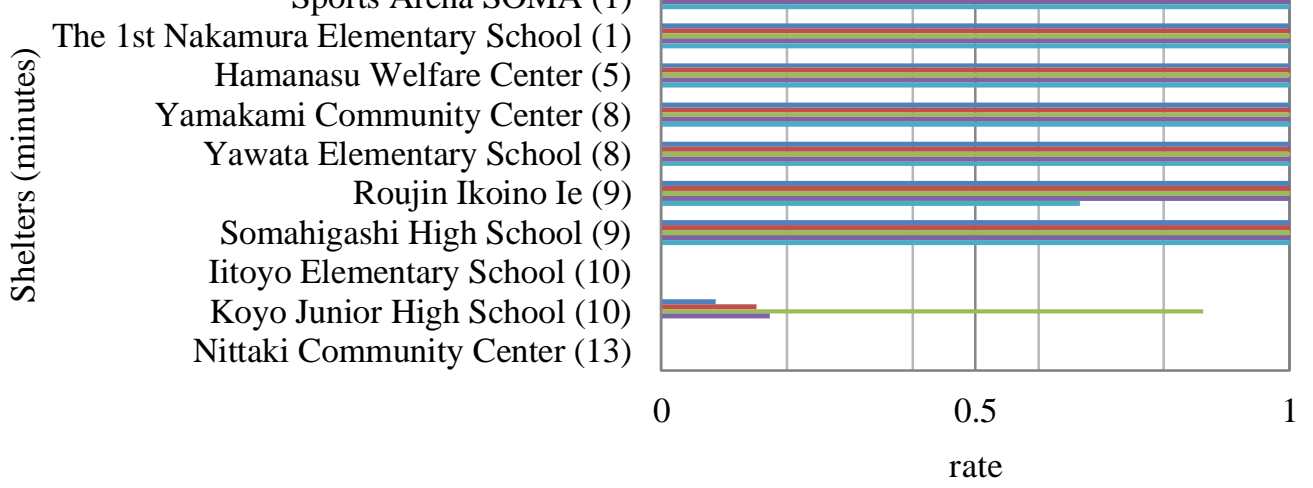

Figure 1 Fulfillment rate (Efficacy) 


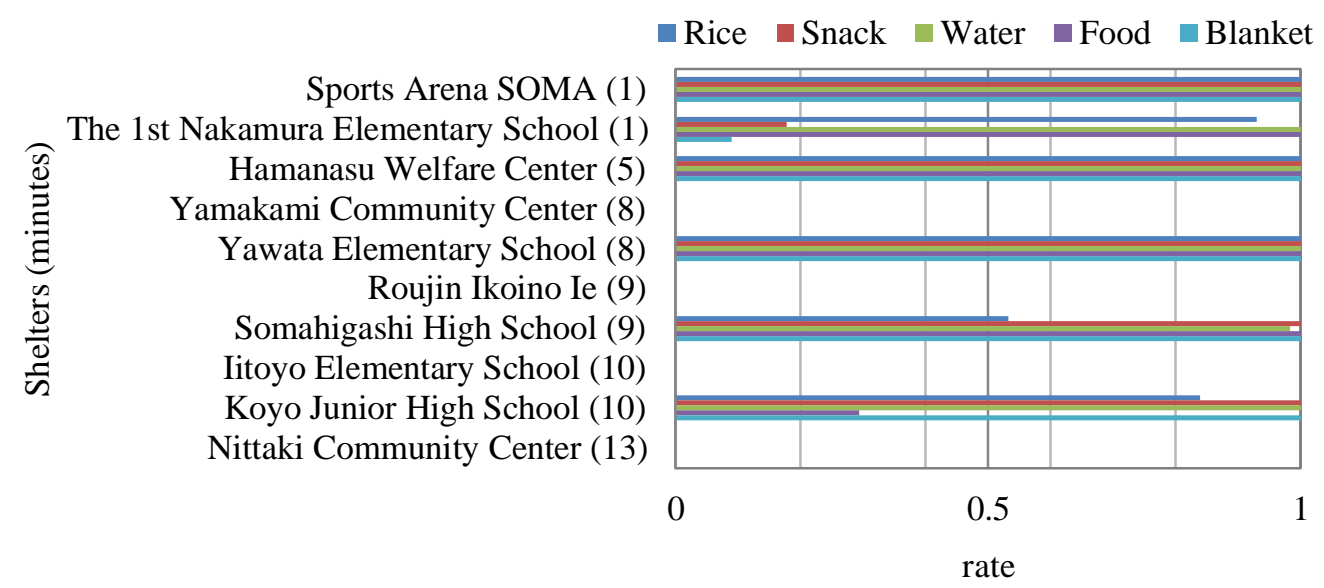

Figure 2 Fulfillment rate (Efficiency)

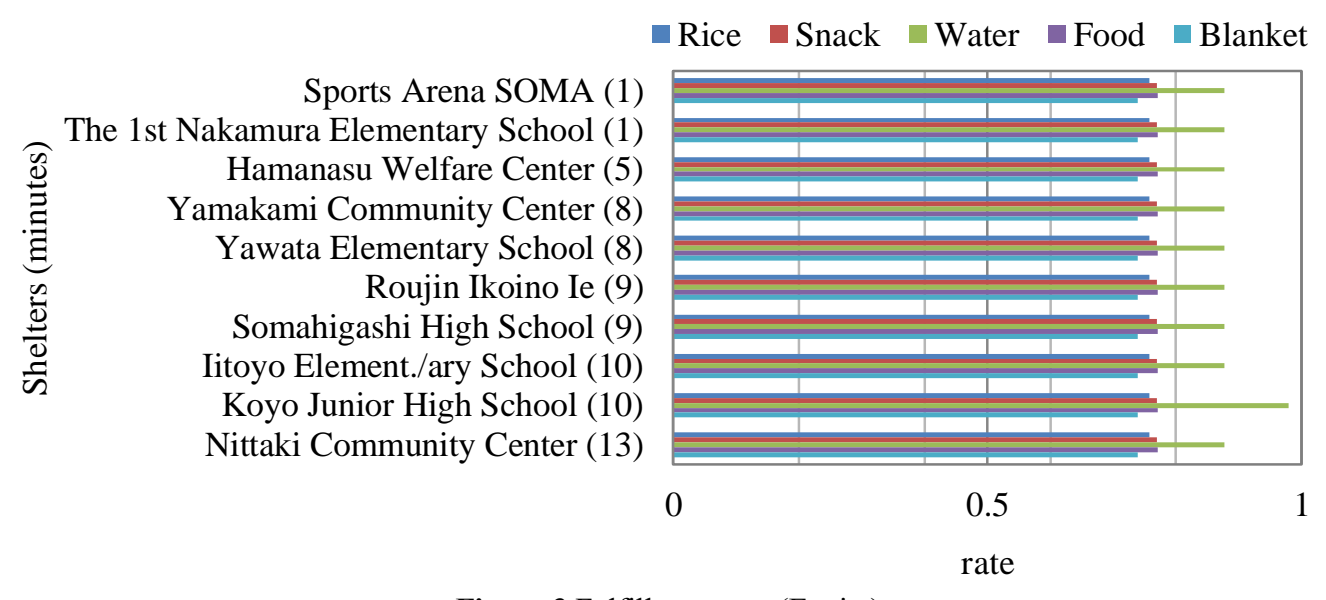

Figure 3 Fulfillment rate (Equity)

\section{DISCUSSION}

Figures 1 to 3 indicate fulfillment rates that facilitate understanding of the differences between efficacy, efficiency, and equity. First, from the result of efficiency (Figure 2), the amount of relief goods is biased because of consideration only for the minimization of distribution costs. On the other hand, from the result of efficacy (Figure 1), a bias existed towards the shelters close to second stockyard. This can be considered to be a result of efficacy, in essence minimizing the value of undelivered relief goods during distribution time. Therefore, compared to the efficiency results, the efficacy results considered not only the whole distribution time, but also delivery.

Next, according to the results of equity (Figure 3), by the metric of equity, the fulfillment rates of relief goods at each shelter were all equal with the exception of one. Although there was a different part of fulfillment rate, this was due to the number of available vehicles. From the results of equity, this was the metric that equitable distribution considered.

\subsection{Characteristics of the Proposed Efficacy Metric}

Based on the results from the numerical experiments, to show the characteristics of the efficacy metric, we examined the shortage amount per time unit for each metric using the objective function values of the cases applying the optimal solutions by the metric of efficiency and equity to the objective function of the metric of efficacy. The results are shown in Table 2.

The objective function value of the optimal solution of the efficacy metric was 37.557. On the other hand, the results that applied the optimal solution of the efficiency and equity metrics to the objective function of the efficacy metric were 44.938 and 144.573, respectively. From these results, we found that the metric of efficacy is not completely covered by metric of the efficiency and equity with regard to effective utilization of distribution time.

\subsection{Multi-objective Evaluation}

In a disaster, in fact more than one metric is important in supplying goods right after the disaster. Furthermore, importance of those metrics is assumed to change overtime after the disaster. In this study efficacy and equity can be 
captured as the metrics that should be considered aftermath of a disaster. Therefore, we carry out numerical experiments of multi-objective evaluation by weighting of efficacy and equity.

By using metric index and parameters of the optimal value, the worst value and weight of each metric, we minimize the objective function using equation (13).

Index

$n \quad$ Metric index, $n=1,2$

\section{Parameters}

$f_{n}^{+} \quad$ The optimal value of metric $n$

$f_{n}^{-} \quad$ The worst value of metric $n$

$\alpha \quad$ The weight value of efficacy, $\alpha=0.1,0.2, \ldots, 0.9$

$$
\begin{aligned}
& \text { Efficacy } \quad f_{1}=\sum_{i} \sum_{p}\left(d_{i p}-x_{i p}\right) \frac{1}{t_{i}} \\
& \text { Equity } \\
& f_{2}=\sum_{p}\left(1-m f_{p}\right)
\end{aligned}
$$

$$
\text { Minimize } \quad \alpha \frac{f_{1}-f_{1}^{+}}{f_{1}^{-}-f_{1}^{+}}+(1-\alpha) \frac{f_{2}-f_{2}^{+}}{f_{2}^{-}-f_{2}^{+}}
$$

Statement (13) is the objective function that represents multi-objective evaluation. The constraints are the same as those given in equations (2) through (7) as well as equation (10).

The results of numerical experiments based on multiobjective evaluation when the value of $\alpha$ is 0.1 and 0.9 are shown in Figure 4 and Figure 5, respectively. Figures 4 and 5 show the fulfillment rates indicating the rations of the amount of delivery to the amount of demand. Additionally, the standardized values of the objective function values of efficacy and equity are shown in Figure 6.

First, Figures $\mathbf{4}$ and $\mathbf{5}$ indicate fulfillment rates that facilitate understanding of the differences of the values of $\alpha$. By these figures, decrease of minimum fulfillment rate is verified by decrement of weight of the metric of equity that indicates the value of $1-\alpha$.

Next, according to Figure 6, the tendency of variation in case of corresponding efficacy and equity simultaneously is indicated by changing the values of $\alpha$ respectively. It is conceivable that this result shows how much the metric of efficacy and equity should be considered simultaneously.

Table 2 The Objective Function Values of the Metric of Efficacy

\section{The objective function value of the metric of efficacy} (Shortage amount / min)

\begin{tabular}{lc}
\hline The optimal solution of the metric of efficiency & 44.938 \\
The optimal solution of the metric of equity & 144.573 \\
The optimal solution of the metric of efficacy & 37.557 \\
\hline
\end{tabular}

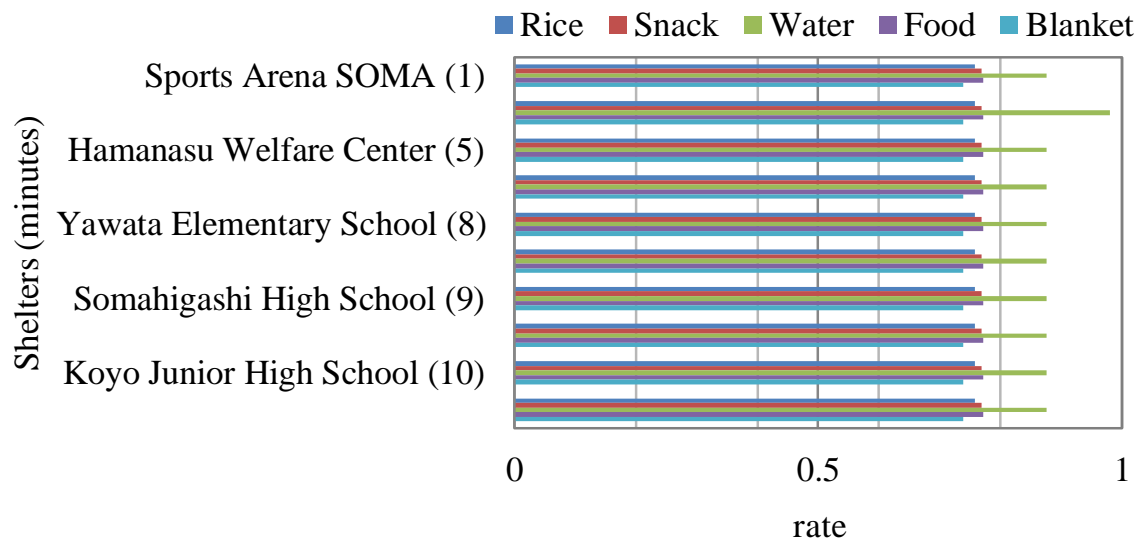

Figure 4 Fulfillment rate $(\alpha=0.1)$ 


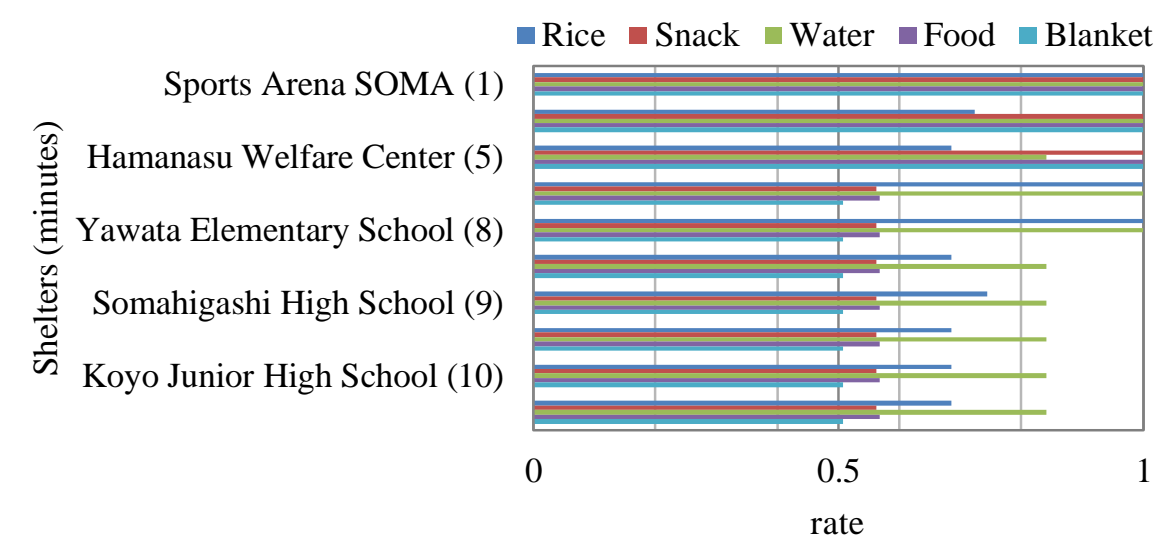

Figure 5 Fulfillment rate $(\alpha=0.9)$

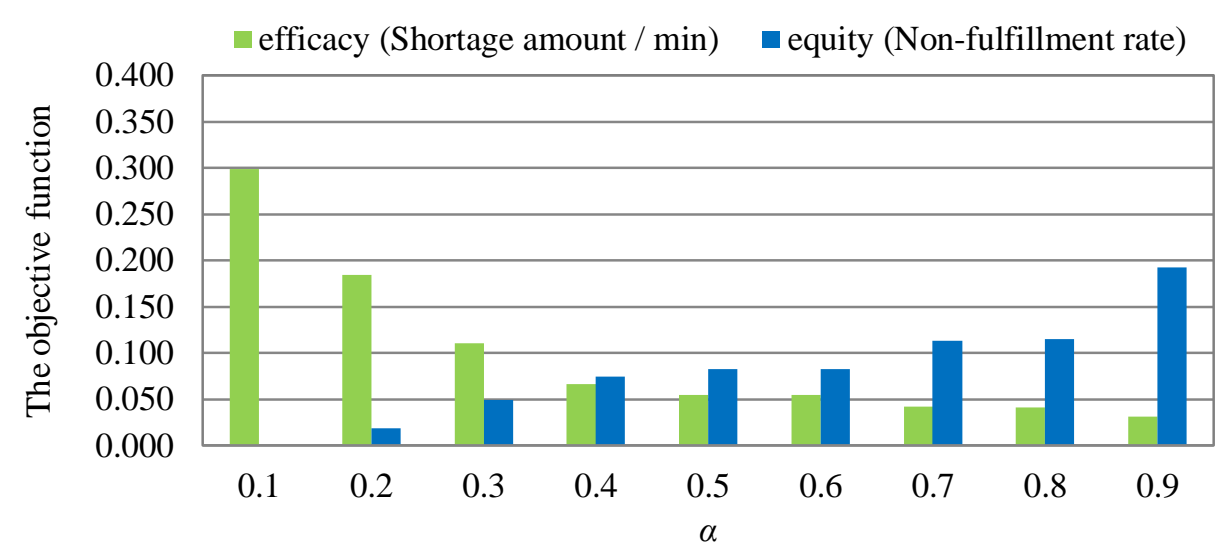

Figure 6 The objective function of each metrics

\section{CONCLUSIONS}

In this paper, we defined efficacy amongst the three metrics of humanitarian logistics. The comparison between the metrics of efficiency, equity, and efficacy was used to identify unique characteristics of the metric of efficacy. As a metric of relief distribution, we proposed "efficacy" to be a measure of effective utilization of distribution time and used numerical experiments to support efficacy as a metric in the context of this definition.

Moreover, we conducted some numerical experiments on the multi-objective evaluation of humanitarian logistics by the means of the metric of equity and the proposed metric of efficacy. From the numerical experiments, we show the relationship of the two metrics by the weight value. It is necessary to consider whether or not an effective distribution is needed in the case of humanitarian aid, in the presence of shelters to which relief goods can be delivered. It is conceivable that the result of the numerical experiments can be used in the decision-making for supply of relief goods considering important degree of the metrics needed for humanitarian aid.

\section{ACKNOWLEDGMENTS}

This research was supported by Grant-in-Aid for Scientific Research (c), number 26350428 from the Japan Society for Promotion of Science (JSPS).

\section{REFERENCES}

Apte, A. (2009). Humanitarian logistics: a new field of research and action, Foundations and Trends in Technology, Information and Operations Management, 3(1), pp. 1-100.

Balcik, B., Beamon, B. M. and Smilowitz, K, S. (2008). Last mile distribution in humanitarian relief, Journal of Intelligent Transportation Systems, 12 (2), pp. 51-63.

Bealt, J., Barrera, J. C. F., and Mansouri, S. A. (2016).Collaborative relationships between logistics service providers and humanitarian organizations during disaster relief operations, Journal of Humanitarian Logistics and Supply Chain Management, 6 (2), pp. 118-144.

CILT (2013). Feasibility of establishing a humanitarian logistics hub in Hong Kong, available at http://member.cilt.org.hk/webadmin/img/news/361_1. pdf, (Accessed: 18 March 2016).

Chakravarty, A. K. (2014). Humanitarian relief chain: rapid response under uncertainty, International Journal of Production Economics, 151, pp. 146-157.

Chou, J.S., Tsai, C.F., Chen, Z.Y. and Sun, M.H. (2014). Biological-based genetic algorithms for optimized disaster response resource allocation, Computers \& Industrial Engineering, 74, pp. 52-67.

Diaz, R., Kumar, S. and Behr, J. (2015). Housing recovery in the aftermath of a catastrophe: Material resources perspective, Computers \& Industrial Engineering, 81, pp. 130-139. 
Haghani, A. and Oh, S. C. (1996). Formulation and solution of a multi-commodity, multi-modal network flow model for disaster relief operations, Transportation Research Part A, 30 (3), pp. 231-250.

Holguin-Veras, J., Taniguchi, E., Jaller, M., Aros-Vera, F., Ferreira, F. and Thompson, R. G. (2014). The Tohoku disasters: Chief lessons concerning the post disaster humanitarian logistics response and policy implications, Transportation Research Part A, 69, pp.86-104.

Hoyos, M.C., Morales, R.S. and Akhavan-Tabatabaei, R. (2015). OR models with stochastic components in disaster operations management: A literature survey, Computers \& Industrial Engineering, 82, pp. 183-197.

Huang, K., Jiang, Y., Yuan, Y., and Zhao, L. (2015). Modeling multiple humanitarian objectives in emergency response to large-scale disaster, Transportation Research Part E, 75, pp. 1-17.

Huang, M., Smilowitz, K., and Balcik, B. (2012). Models for relief routing: equity, efficiency and efficacy, Transportation Research Part E, 48, pp. 2-18.

Kabata, M., and Kainuma, Y. (2013). Research on disaster relief operation; a case of the Great East Japan Earthquake, Journal of Japan Industrial Management Association, 64 (3), pp. 480-487.

Kato, Y., and Kubo, M. (2012). A mathematical approach for final delivery in humanitarian logistics, Proceedings of Scheduling Symposium 2012, pp. 155-160.

L'Hermitte, C, Bowles M., Tatham, P. and Books, B. (2015).An integrated approach to agility in humanitarian logistics, Journal of Humanitarian Logistics and Supply Chain Management, 5 (2), pp. 209-233.

Lin, Y-H, Batta, R., Rogerson, P. A., Blatt, A. and Flanigan, M. (2011). A logistics model for emergency supply of critical items in the aftermath of a disaster, SocioEconomics Planning Science, 45, pp. 132-145.

Özdamar, L. and Demir, O. (2012). A hierarchical clustering and routing procedure for large scale disaster relief logistics planning, Transportation Research Part E:
Logistics and Transportation Review, 48 (3), pp. 591602.

Özdamar, L. and Ertem, M.A. (2015). Models, solutions and enabling technologies in humanitarian logistics, European Journal of Operational Research, 244 (1), pp. 55-65.

Özdamar, L., Ekinci, E. and Küçükyazici, B. (2004). Emergency logistics planning in natural disaster, Annals of Operations Research, 129, pp. 217-245.

Pujawan, I. N., Kurniati, N. and Wessiani, N. A. (2009). Supply chain management for disaster relief operations: principles and case studies, International Journal Logistics Systems and Management, 5 (6), pp. 679-692.

Rath, S. and Gutjahr, W. J. (2014). A math-heuristic for the warehouse location-routing problem in disaster relief, Computers \&Operations Research,42, pp. 25-39.

Rezaei-Malek, M., Tavakkoli-Moghaddam, R., Zahiri, B. and Bozorgi-Amiri, A. (2016).An interactive approach for designing a robust disaster relief logistics network with perishable commodities, Computers \& Industrial Engineering, 94, pp. 201-215.

Soeta, H., Kabata, M. and Kainuma, Y. (2015). Development of logistics model for disaster relief operations, Journal of Japan Industrial Management Association, 66 (1), pp. 23-29.

Tomasini, R. M. and Van Wassenhove, L. N. (2009). From preparedness to partnership: case study research on humanitarian logistics, International Transaction in Operational Research, 16, pp. 549-559.

Tzeng, G. H., Cheng, H. J. and Huang, T. D. (2007). Multiobjective optimal planning for designing relief delivery systems, Transportation Research Part E, 43, pp. 673686.

Xu, 1. and Beamon, B. M. (2006). Supply chain coordination and cooperation mechanisms: an attribute-based approach, Journal of Supply Chain Management: A Global Review of Purchasing \& Supply, 42 (1), pp. 412.

Takeo Kobayashi is an expert of production management at RICOH COMPANY LTD. He received his M.Sc. degree from Tokyo Metropolitan University, Tokyo, Japan in 2017, supervised by Prof. Y. Kainuma. His research interests include disaster relief operations, humanitarian logistics and optimization.

Yacob Khojasteh is an Associate Professor of Operations Management at the Graduate School of Global Studies, Sophia University, Japan. He received his Ph.D. in Engineering from the University of Tsukuba, Japan. His recent books entitled "Supply Chain Risk Management", and "Production Management" were published by Springer and CRC Press, respectively. His research interests include production and operations management, supply chain management, system modeling and optimization, and lean production system.

Yasutaka Kainuma is currently a Professor of Operations Management at the Department of System Design, Tokyo Metropolitan University, Japan. He received his Ph.D. from School of Science and Technology, Meiji University, Japan. He is the President of Japanese Operations Management and Strategy Association, the Vice President of Japan Industrial Management Association, and the Board of Trustees Member of the International Foundation for Production Research - Asia Pacific Region. His research interests include supply chain management, closed-loop supply chain, sustainable operations, remanufacturing and humanitarian logistics. He won the best paper award of the $21^{\text {th }}$ ICPR (2011), the outstanding paper award of the $14^{\text {th }}$ APIEMS Conference (2013) and $17^{\text {th }}$ APIEMS. 